\title{
Quality of life in obese patients after thyroidectomy for goiter
}

\author{
Courtney Brooke Shires ${ }^{1}$, Nic Beckmann ${ }^{2}$, Theodore Klug $^{1}$, John D. Boughter Jr ${ }^{3}$ \\ ${ }^{1}$ West Cancer Center, Germantown, TN, USA; ${ }^{2}$ Colorado ENT \& Allergy, Colorado Springs, CO, USA; ${ }^{3}$ Department of Anatomy and \\ Neurobiology, University of Tennessee Health Science Center, Memphis, TN, USA \\ Contributions: (I) Conception and design: All authors; (II) Administrative support: All authors; (III) Provision of study materials or patients: All \\ authors; (IV) Collection and assembly of data: All authors; (V) Data analysis and interpretation: All authors; (VI) Manuscript writing: All authors; (VII) \\ Final approval of manuscript: All authors. \\ Correspondence to: Courtney Brooke Shires, MD. 7945 Wolf River Blvd, Germantown, TN 38104, USA. Email: cshires1@gmail.com.
}

\begin{abstract}
Background Compressive symptoms are common in thyroid disease. Many studies have focused on the size of the gland and its effects on patients. However, few have taken into account the body mass of the patient. The aim of our study was to examine whether or not a patient's body mass index (BMI) influences symptomatic outcomes following thyroid surgery for benign disease.

Methods: We conducted a prospective analysis evaluating 60 patients that underwent thyroidectomy for benign goiter (single or multinodular) disease. Patients were classified as obese, overweight, or normal based on BMI. Pre- and post-operative surveys were administered including the MRC breathlessness scale, M.D. Anderson Dysphagia Inventory (MDADI), and the ThyPRO quality of life questionnaire to evaluate dysphagia, dyspnea, and quality of life respectively.

Results: Patients classified as obese $(n=37)$ scored significantly worse pre-operatively on MRC, MDADI, and ThyPRO surveys when compared to overweight $(n=13)$ or normal weight $(n=10)$ counterparts. Subjects in the obese group, but not the other groups, showed post-surgical improvement on both the MRC and MDADI surveys $(\mathrm{P}<0.0001)$. Similarly, obese subjects showed significant improvement on all 11 domains of the ThyPRO survey following surgery $(\mathrm{P}<0.0001)$, and overall degree of improvement was highly correlated with $\mathrm{BMI}$ among all subjects $(\mathrm{r}=0.60 ; \mathrm{P}=0.0005)$.
\end{abstract}

Conclusions: Obesity, as determined by BMI, is a critical factor to consider in the alleviation of compressive symptoms before and after thyroidectomy for goiter. Our analysis of survey data indicates obese subjects have increased benefit of surgery compared to their lighter counterparts.

Keywords: Thyroid; thyroidectomy; goiter; benign thyroid disease; nodule; malignancy; obesity; dysphagia; dyspnea

Submitted Apr 04, 2020. Accepted for publication Feb 23, 2021.

doi: $10.21037 / g s-20-441$

View this article at: http://dx.doi.org/10.21037/gs-20-441

\section{Introduction}

Benign thyroid disorders and goiter are not an uncommon occurrence in the population and can lead to significant patient complaints. In population studies of iodine sufficient areas, goiter incidence ranges from $1 \%$ to $5.9 \%$ $(1,2)$. Nearly $80 \%$ of nodular thyroid disease is caused by hyperplasia of the gland and this occurs in up to $5 \%$ of any population (3). This etiology can include iodine deficiency, disorders of hormonogenesis, and poor utilization of iodine amongst many others (1-6). When this growth leads to an increase in the size of the gland it is termed a goiter (7). Oftentimes these patients will present secondary to enlarging mass of the neck noticed by the patient or primary provider. The goiter can be without a nodule, uni-nodular,

^ ORCID: 0000-0002-2829-5846. 
or multi-nodular and likewise the patient can be euthyroid, hypo, or hyper-thyroid $(1-3,7,8)$.

Patients with goiter often present with compressive complaints of difficulty swallowing, cough, and respiratory changes. Luo showed that $61 \%$ of patients with benign multinodular goiter have compressive symptoms (9). Dysphagia has been associated with goiter size and substernal extension of goiter (10). This has been related to direct compression and can cause worsening dysphagia to solids (1). In a study of 273 consecutive goiter patients, two thirds had symptoms of significant dyspnea, dysphagia or both after presenting with benign goiter (11). Greenblatt et al. (in 2009) used a swallowing quality of life questionnaire (SWAL-QOL) and found that 8/11 domains in this inventory improved following uncomplicated thyroidectomy (10).

Dyspnea is also a common complaint of patients with goiter. A previous study found that $59 \%$ of patients with substernal extension of their goiter complained of dyspnea (12). Yip et al. (in 2014) evaluated dyspnea in patients receiving thyroidectomy, and reported that $82.4 \%$ of cases overall had improvement, with $65 \%$ occurring in cervical goiter and increasing to $88 \%$ if noted to have substernal extension (13). Furthermore, this increased to $97.9 \%$ if total gland size was greater than 100 grams or $100 \%$ if greater than 75 grams for single lobe.

In addition to compressive symptoms, overall quality of life has also been studied in patients with goiters. There has been a growing interest within Thyroidology in measuring patient reported outcomes (PRO), leading to the development of a comprehensive PRO survey measuring thyroid-related quality of life, the ThyPRO $(14,15)$. Using this survey, quality of life was shown to improve in response to treatment (including thyroidectomy) across the range of benign thyroid disease $(8,14)$. In one of these studies, Mishra and colleagues (in 2013) found that the weight of the goiter did not have a significant effect on quality of life scores (8). However, body weight or body mass index (BMI) of the patients was not examined. There is evidence that BMI correlates with thyroid size, and in males, obesity is associated with greater thyroid volume and more frequent occurrence of nodules $(4,16)$.

In the current study, we investigated whether obesity impacts functional recovery following thyroidectomy, including improvement of compressive symptoms and PRO quality of life. To do this, we stratified patients according to BMI, and evaluated dysphagia, dyspnea and quality of life using pre- and post-operative surveys, including the
M.D. Anderson Dysphagia Inventory (MDADI), Medical Research Council (MRC) breathlessness scale, and the ThyPro quality of life questionnaire.

We present the following article in accordance with the STROBE reporting checklist (available at http://dx.doi. org/10.21037/gs-20-441).

\section{Methods}

\section{General treatment}

The study was conducted according to the guidelines of the Declaration of Helsinki (as revised in 2013), and (pilot phase) approved by the Institutional Review Board (IRB) of University of Tennessee (Code 18-06423-XP). This study was a prospective analysis evaluating 60 patients that underwent thyroidectomy for benign goiter (single or multinodular) disease. Thyroidectomy was defined as either hemithyroidectomy with/without isthmusectomy or total thyroidectomy. Data was collected from January 2016 to August 2018. The study included all patients undergoing thyroid surgery for goiter and benign lesions. Patients were excluded if any nodules within the thyroid returned positive for malignancy. All study subjects provided informed consent.

Overall patient characteristics, including BMI, age, sex and thyroid volume, were recorded and listed in Table 1.

\section{Study design}

Pre- and post-operative surveys were performed including MDADI, MRC breathlessness scale, and the ThyPRO quality of life questionnaire to evaluate dysphagia, dyspnea, and quality of life respectively. The MDADI questionnaire has been validated in head and neck cancer patients to evaluate quality of swallowing after treatment; this tool includes global, emotional, functional and physical subscales (17). The MRC breathlessness scale quantifies the disability associated with breatblessness by identifying that breatblessness occurs when it should not (Grades 1 and 2) or by quantifying the associated exercise limitation (Grades 3-5). The score is the number that best fits the patient's level of activity (18). The current 85 -item version of ThyPRO consists of 13 scales, covering domains of physical (4 scales) and mental (2 scales) symptoms, function and well-being ( 3 scales) and participation/social function (4 scales) $(14,15)$. The surveys were given at the initial pre-operative appointment and then at the 6-month 
Table 1 Patient characteristics

\begin{tabular}{lcccccc}
\hline Group & $\mathrm{n}$ & $\mathrm{BMI}$, range & BMI, median & Age $($ mean \pm SD) & Sex $(\% \mathrm{~F})$ & Thyroid volume $(\mathrm{mean} \pm \mathrm{SD})$ \\
\hline Obese & 37 & $30.1-74.2$ & 39.9 & $46.5 \pm 12.1$ & 89 & $97.9 \pm 121.8$ \\
Overweight & 13 & $25.0-29.4$ & 28.3 & $50.5 \pm 17.5$ & 92 & $56.8 \pm 80.8$ \\
Normal & 10 & $20.4-24.9$ & 24.0 & $45.0 \pm 15.8$ & 90 & $49.8 \pm 66.1$ \\
Total & 60 & & & $47.1 \pm 13.9$ & 90 & $81.0 \pm 107.3$ \\
\hline
\end{tabular}

$\mathrm{BMI}$, body mass index. \%F, percentage that are female.

follow-up.

\section{Statistical analysis}

Total thyroid volumes were calculated for each surgery using the accepted ellipsoid formula of 0.524 times height, width, and depth of each lobe (9).

To examine the possible relationship between BMI and thyroid volume, as well as performance on the MDADI, MRC and ThyPRO scales, we compared data separated into groups by BMI using standard metrics, i.e., obese $(>0.30)$, overweight (range, 25-29.4) and normal (range, 20.4-24.9). Most (54/60) subjects were female, and this proportion (89$92 \%$ ) did not differ significantly across the BMI groups (as determined by Fisher's exact test).

We recorded patient insurance type. For examining the effects of insurance type on survey results, responses were omitted from 2 subjects that reported having no insurance.

Thyroid volumes were log-transformed and analyzed according to group (either BMI or insurance) with one-way ANOVAs. The relationship between individual BMI and thyroid volume was also evaluated with correlation analysis.

The MRC breathlessness survey is a single 5-grade scale; therefore, responses either pre- or post-surgery were treated as an ordinal variable, and statistical analyses were non-parametric. Group differences for MRC were analyzed with Kruskal-Wallace H test, including Dunn's post-hoc test. Pre-and-post comparisons within groups were made using the Wilcoxon matched-pairs rank test.

For the MDADI and ThyPRO surveys, pre- and postsurgical Likert scale data were averaged and linearly transformed to a range of 0-100. Therefore, ANOVA was appropriate for analysis of group differences, either according to BMI or insurance type (including Tukey's test for post-hoc comparisons). These included one-way ANOVAs for MDADI score, either pre- or post.

Two-way (group vs. test domain) mixed ANOVAs were used to examine ThyPro scores, including pre-, post, or their difference. Paired $t$-tests were used for additional within-group pre-and post-comparisons.

Since the BMI groups differed in size (Table 1), we also examined the relationship between individual BMI score and survey results using correlation analysis. For all measures (i.e., thyroid volume and surveys), we performed additional, similar analyses to examine the potential effects of insurance type (responses were omitted from 2 subjects that reported having no insurance). There was no effect of insurance type on BMI (one-way ANOVA), and significant interactions between BMI group or insurance type for MDADI or ThyPro were not detected using additional multi-factor ANOVAs. Finally, pairwise correlations on MRC, MDADI, and ThyPRO values was performed to examine whether or not individual patients tended to show consistent improvements across scales. All statistical analyses were conducted using SPSS v. 25 (IBM Corp. Armonk, NY, USA).

\section{Results}

\section{Distribution of patients}

Among all subjects $(\mathrm{n}=60), 29$ underwent total thyroidectomy, 15 left lobectomy, and 16 right lobectomy. The majority of patients had their thyroid removed for multinodular goiter (47\%) and benign goiter (20\%), with $15 \%$ found to have adenomatous nodule and $15 \%$ with thyroiditis upon pathology review. Substernal extension was noted in $38(63 \%)$ patients.

\section{Thyroid volume}

Total volume range was 0.63 to $630 \mathrm{~mL}$ with an average of $81 \mathrm{~mL}$ (Table 1). Obese subjects had a higher mean volume than the other two groups, although this measurement was in general highly variable among subjects. There was 


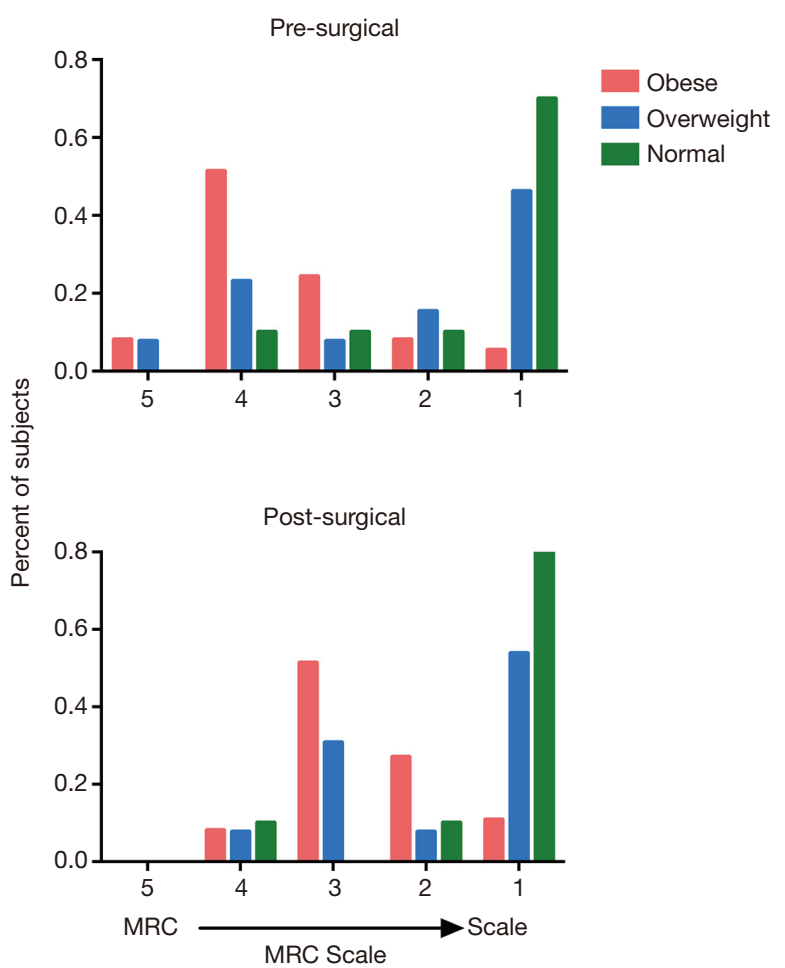

Figure 1 MDADI scores before (Pre-op) or following surgery (Post-op). (A) Mean scores ( \pm SEM) compared among BMI groups. Means labeled a-b are significantly different from one another. (B) Individual scores grouped into columns based on insurance types. Individual symbols are color-coded based on BMI category. Column means are denoted by horizontal bars. Although patients with Medicaid tend to have significantly lower MDADI scores than those with Medicare or private insurance, the distribution of BMI types within each insurance group shows that these two factors are not consistently related. BMI, body mass index; MDADI, M.D. Anderson Dysphagia Inventory.

a small yet significant effect of BMI group $\left(\mathrm{F}_{[2,59]}=4.43\right.$, $\mathrm{P}=0.016$ ), but not insurance type, with obese subjects $>$ normal subjects (but not overweight subjects). There was a moderate correlation between BMI and thyroid volume across all subjects (Pearson $r=0.32, \mathrm{P}=0.012$ ).

\section{Insurance}

Type of insurance was also considered as a factor. Each subject was classified as having one of four types of coverage (\% for all 60 subjects): Medicaid (46.7\%), Medicare (16.7\%), private $(33.3 \%)$, or none $(<1 \%)$. Within BMI groups, these proportions were roughly similar. Accordingly, results of one-way ANOVAs indicated no effect of insurance type (excluding those with no insurance, as this group contained only 2 patients) on either BMI or thyroid volume.

\section{$M D A D I$}

Prior to surgery, obese patients had a lower (worse) average score on the MDADI scale, relative to overweight or normal weight subjects (Figure 1A). There was a significant effect of group on pre-surgical MDADI scores $\left(\mathrm{F}_{[2,57]}=\right.$ $12.12, \mathrm{P}<0.0001)$, with obese subjects different from both overweight and normal subjects. Following surgery, subjects in the obese group (but not the other two groups) saw significant improvement $(\mathrm{P}<0.0001)$. Following surgery, there still was a main effect of group $\left(\mathrm{F}_{[2,57]}=6.95, \mathrm{P}=0.002\right)$ with only the obese $v s$. overweight difference significant in the posthoc test. Both pre- and post-surgical MDADI scores showed small-moderate negative correlations with BMI (Pearson $r s>-0.26$, ps $<0.048$ ).

Interestingly, MDADI score varied among insurance groups (Figure $1 B)$, both pre- $\left(\mathrm{F}_{[2,57]}=8.98(\mathrm{P}=0.0004)\right.$ and post-surgery $\left(\mathrm{F}_{[2,57)}=5.68, \mathrm{P}=0.006\right)$. Subjects with Medicaid were significantly more likely to have lower scores on this scale than subjects with either Medicare or private insurance.

\section{MRC breatblessness scale}

Prior to surgery, obese patients scored significantly worse (i.e., a higher score) on the MRC scale than either overweight or normal subjects $\left[\chi^{2}(2)=17.12, \mathrm{P}=0.0002\right]$. However, subjects in the obese group showed substantial improvement following surgery (Figure 2), as evidenced by significantly lower MRC scores following surgery $(\mathrm{P}<0.0001)$. The degree of improvement in the other two groups was not significant, as both of these groups already had a majority of their subjects in the category of lowest (best) score prior to surgery. Despite the improvement by obese subjects, a significant group difference in MRC scores persisted after surgery $\left[\chi^{2}(2)=12.7, \mathrm{P}=0.0018\right]$, with obese subjects > normal (but not overweight) subjects. As expected from these results, both MRC pre- and post-surgical score correlated strongly with BMI (Spearman $r \mathrm{~s}>0.50$, $\left.P_{\mathrm{S}}<0.0001\right)$.

\section{ThyPRO quality of life survey}

As shown in Figure $3 A$, obese subjects had significantly 

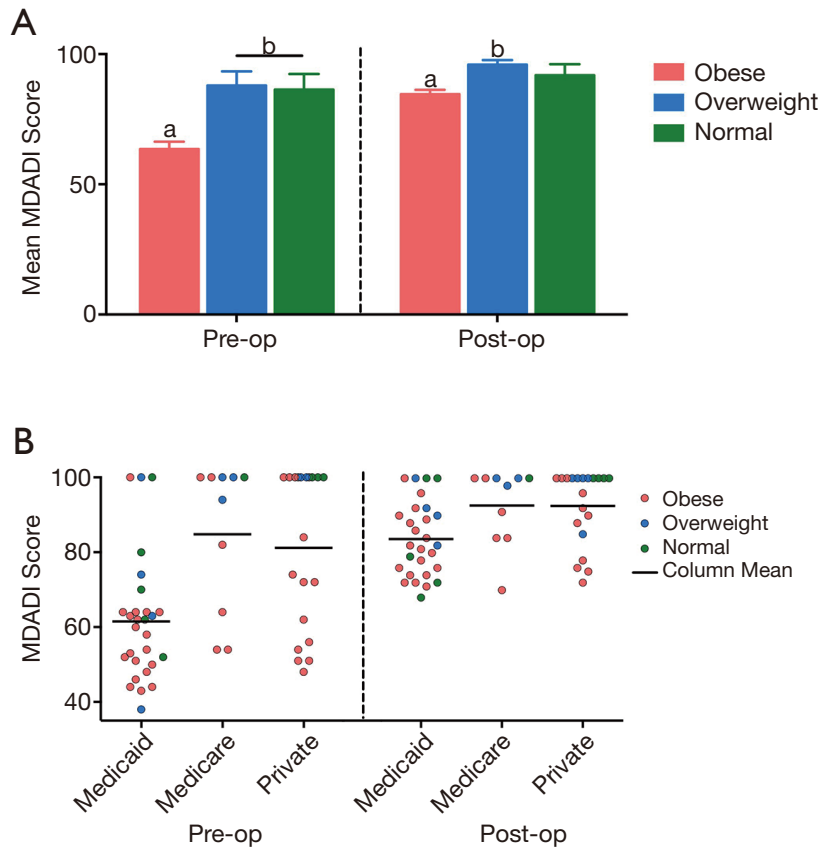

Figure 2 Frequency distribution of MRC scores among obese, overweight, and normal subjects. Scores for obese subjects improved following surgery. MRC, Medical Research Council

higher (worse) scores on the 12-domain ThyPRO survey pre-operatively compared to overweight or normal subjects $\left(\mathrm{F}_{[2,57]}=16.52, \mathrm{P}<0.0001\right)$. Post-operatively (Figure 3B), the effect of group was no longer significant $\left(\mathrm{F}_{[2,57]}=2.37\right.$, $\mathrm{P}=0.102)$. When the degree of improvement was visualized (Figure 3C), it was clear that obese subjects improved across the board on all domains of the survey $\left(\mathrm{F}_{[2,57]}=27.15\right.$, $\mathrm{P}<0.0001)$. Due to consistency across domains, an average "master" ThyPRO improvement score was calculated for use in correlation analysis. This score was strongly correlated with BMI (Pearson $r=0.60 ; \mathrm{P}=0.0005$ ).

As was the case with MDADI results, insurance type had an effect on the ThyPRO scores, both in terms of average pre-surgical score $\left(\mathrm{F}_{[2,5]]}=8.642, \mathrm{P}=0.001\right)$ and improvement score $\left(\mathrm{F}_{[2,57]}=6.054, \mathrm{P}=0.004\right)$. Patients with Medicaid had higher (worse) preliminary scores than those with Medicare or Private insurance, and correspondingly a greater degree of improvement following surgery.

Finally, pairwise correlations on MRC, MDADI, and ThyPRO scales (both pre-and post-surgery) show these subjective measures are consistently related in individual subjects (Spearman's $r$ s range from 0.32 to $0.68, P \mathrm{~s}<0.003$ ),
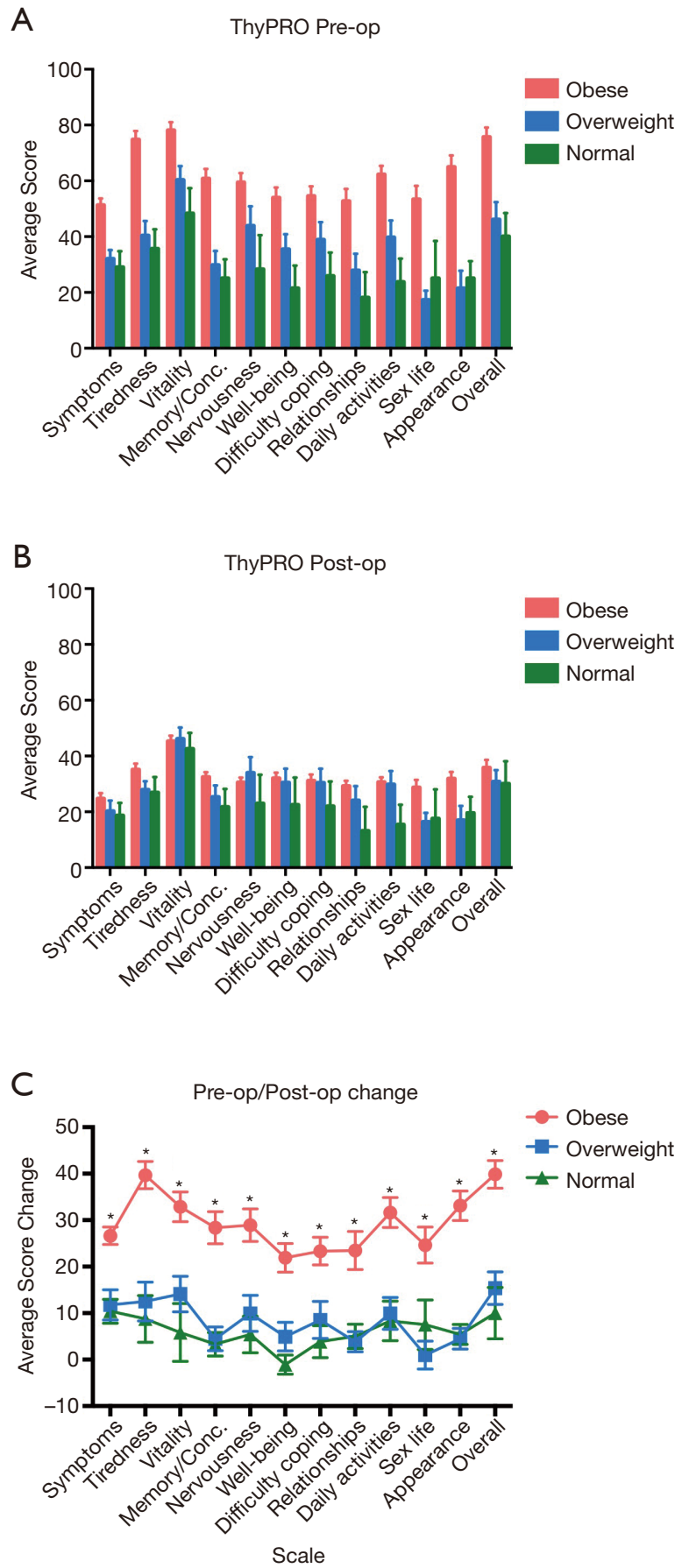

Figure 3 Mean ThyPRO scores before (A) and following surgery (B). When the degree of improvement is analyzed (C), obese subjects show significant improvement on every scale relative to the other two groups. Asterisks in (C) denote significant differences (Tukey's posthoc test; $\mathrm{P}<0.01$ ) between obese and the two other groups of subjects. *, $\mathrm{P}<0.05$. ThyPRO, patient reported outcomes survey measuring thyroid-related quality of life. 
i.e., patients reporting positive performance on any one scale tended to report positive performance on the others.

\section{Discussion}

It is established that obesity is associated with changes in thyroid function, and there is evidence that in certain populations, obesity may be a factor in the likelihood of developing goiter (4,16,19-22). Furthermore, obesity is commonly associated with gastroesophageal reflux, which in turn is a well-known cause of dysphagia $(23,24)$. Indeed, in our study obese patients had lower preoperative MDADI scores as compared to overweight or normal weight subjects, indicating poorer swallowing function. Following thyroidectomy, obese patients showed considerable improvement as reflected by increased MDADI scores. Greenblatt and colleagues (in 2009) estimated swallowingrelated quality of life following thyroid surgery with a self-administered questionnaire, the SWAL-QOL (10). Results of this survey indicated that thyroid surgery led to significant perceived improvements in many aspects of swallowing-related quality of life, similar to our MDADI results. In our study, overweight and normal weight groups did not see the same degree of improvement in swallowing as did obese subjects. However, a number of patients had max scores pre-operatively and thus could not improve further; there were proportionally more of these in the nonobese groups.

We also found that obese patients had more problems with breathlessness, as indicated by lower MRC scores. The relationship between obesity and dyspnea is firmly established, although the specific mechanisms are not completely understood (25). Post-operatively, obese subjects in our study had a significantly greater improvement compared to the other groups. Furthermore, BMI was significantly correlated with pre- and post-op MRC scores, as well as magnitude of improvement, across all subjects. This is interesting, as we did not perform any pulmonary/ cardiac rehabilitation after surgery. The patients also did not have a significant weight loss after surgery.

Multiple studies have shown improved quality of life after surgery for hyper or hypo-functioning thyroid glands $(26,27)$. Improved quality of life has also been shown after removing large benign euthyroid symptomatic goiters $(8,14,15,28)$. However, the benefit thyroidectomy has based on patient body weight has not been investigated. It is generally accepted that quality of life is negatively influenced by thyroid dysfunction (29). Tabriz et al.
(2020) found that $86 \%$ of patients had no change or improved quality of life after hemithyroidectomy or total thyroidectomy for symptomatic benign goiter (30). The results of the ThyPRO survey in our study showed that obese subjects had significantly worse scores across all 12 domains preoperatively, with normalizing post-operatively. Postoperatively, however, these patients had significant improvement in quality of life in all 12 domains.

In general, patients belonging to a lower socioeconomic demographic show minor life satisfaction, more subjective complaints, and a poorer state of health (31). Accordingly, patients with lack of insurance have been shown to present with higher stage diseases (32). Our patients with Medicaid showed had higher (worse) preliminary scores than those with Medicare or Private insurance, and correspondingly a greater degree of improvement following surgery in both MDADI scores and ThyroPro scores. Interestingly, the patients in the Medicaid group seemed to have more perceived improvement in swallowing and quality of life with thyroid surgery than those in the other two insurance groups.

In our study, only 2 ThyPRO domains, vitality and tiredness, were significant when looking at thyroid volume and BMI. This is in contrast to another study that showed anxiety and depression to be statistically significant between study groups. Their study showed the emotional aspect was influenced by thyroid disease. Any intervention, including operation, RAI, or cyst aspiration with ethanol sclerotherapy showed a positive impact in quality of life and anxiety in Tabriz' patients with thyroid disease (30).

We included only euthyroid patients in our study. Mishra included $66 \%$ euthyroid, $31 \%$ toxic, and $3 \%$ hypothyroid patients. In Mishra's study, thyrotoxicosis was the most common indication for thyroid surgery (8). Interestingly, only vitality and tiredness were affected in our study, even though all of our patients were euthyroid both before and after surgery. Mishra also showed an improvement in vitality in their patients no matter the indication for surgery and no matter the preoperative thyroid function. We removed the variable of functionality of the gland in our study and focused on the size of the patient.

Our study shows a higher incidence of thyroid disease in females, which is consistent with several other studies. Women are also more likely to report problems in quality of life than men (30). Mishra et al. (in 2013) found that female gender showed significantly better outcomes in quality of life domains after thyroid surgery. Females seem to benefit more from thyroidectomy than their male counterparts (8). 


\section{Limitations}

Even though the patients perceived a great improvement in several aspects regarding dyspnea, dysphagia, and quality of life, we only conducted subjective tests, not physiological tests or measurements. For example, swallowing may be evaluated pre- or post-surgery using speech therapy evaluation, manometry, videofluoroscopy, modified barium swallow, or endoscopy. Similarly, it would be interesting to directly compare results of the MRC breathlessness scale to formal pulmonary function testing before or after thyroid surgery.

Disorders of sleep, including sleep apnea syndrome and positional dyspnea, would also be interesting to examine in these subject groups. Previous studies showed a positive effect of thyroidectomy on sleep apnea syndrome and positional dyspnea $(33,34)$. Finally, we did not measure neck circumference before or after surgery. Interestingly, Mishra et al. (in 2018) limited their study to patients who had visible goiters, but we did not have that inclusion requirement (8).

\section{Conclusions}

Obese patients score worse pre-operatively across the board in dysphagia, dyspnea, and quality of life surveys when compared to normal or overweight counterparts.

However, when compared post-operatively they have statistically significant improvements in their dyspnea and quality of life scores, regardless of their thyroid volume.

Further multivariate analysis and evaluation of substernal extension is needed but initial analysis seems to indicate that obese patients have some gained benefit from surgery compared to their lighter counterparts.

\section{Acknowledgments}

Funding: None.

\section{Footnote}

Reporting Checklist: The authors have completed the STROBE reporting checklist. Available at http://dx.doi. org/10.21037/gs-20-441

Data Sharing Statement: Available at http://dx.doi. org/10.21037/gs-20-441
Conflicts of Interest: All authors have completed the ICMJE uniform disclosure form (available at http://dx.doi. org/10.21037/gs-20-441). Courtney Brooke Shires serves as an unpaid editorial board member of Gland Surgery from Nov 2018 to Oct 2022. The other authors have no conflicts of interest to declare.

Ethical Statement: The authors are accountable for all aspects of the work in ensuring that questions related to the accuracy or integrity of any part of the work are appropriately investigated and resolved. All study subjects provided informed consent. The study was conducted according to the guidelines of the Declaration of Helsinki (as revised in 2013), and (pilot phase) approved by the Institutional Review Board (IRB) of University of Tennessee (Code 18-06423-XP).

Open Access Statement: This is an Open Access article distributed in accordance with the Creative Commons Attribution-NonCommercial-NoDerivs 4.0 International License (CC BY-NC-ND 4.0), which permits the noncommercial replication and distribution of the article with the strict proviso that no changes or edits are made and the original work is properly cited (including links to both the formal publication through the relevant DOI and the license). See: https://creativecommons.org/licenses/by-nc-nd/4.0/.

\section{References}

1. Sawin CT, Castelli WP, Hershman JM, et al. The aging thyroid. Thyroid deficiency in the Framingham Study. Arch Intern Med 1985;145:1386-8.

2. Tunbridge WM, Evered DC, Hall R, et al. The spectrum of thyroid disease in a community: the Whickham survey. Clin Endocrinol (Oxf) 1977;7:481-93.

3. Hennemann G. Non-toxic goitre. Clin Endocrinol Metab 1979;8:167-79.

4. Dauksiene D, Petkeviciene J, Klumbiene J, et al. Factors Associated with the Prevalence of Thyroid Nodules and Goiter in Middle-Aged Euthyroid Subjects. Int J Endocrinol 2017;2017:8401518.

5. Banks CA, Ayers CM, Hornig JD, et al. Thyroid disease and compressive symptoms. Laryngoscope 2012;122:13-6.

6. Medeiros-Neto G. Iodine deficiency disorders. In: deGroot L, Jameson J. editors. Endocrinology 6th Ed. Chapter 88. New York: Elsevier, 2010. 
7. Solbiati L, Cioffi V, Ballarati E. Ultrasonography of the neck. Radiol Clin North Am 1992;30:941-54.

8. Mishra A, Sabaretnam M, Chand G, et al. Quality of life (QoL) in patients with benign thyroid goiters (pre- and post-thyroidectomy): a prospective study. World J Surg 2013;37:2322-9.

9. Luo J, McManus C, Chen H, et al. Are there predictors of malignancy in patients with multinodular goiter? J Surg Res 2012;174:207-10.

10. Greenblatt DY, Sippel R, Leverson G, et al. Thyroid resection improves perception of swallowing function in patients with thyroid disease. World J Surg 2009;33:255-60.

11. Alfonso A, Christoudias G, Amaruddin Q, et al. Tracheal or esophageal compression due to benign thyroid disease. Am J Surg 1981;142:350-4.

12. Shen WT, Kebebew E, Duh QY, et al. Predictors of airway complications after thyroidectomy for substernal goiter. Arch Surg 2004;139:656-9.

13. Yip L, Stang MT, Carty SE. Thyroid carcinoma: the surgeon's perspective. Radiol Clin North Am 2011;49:463-71, vi.

14. Watt T, Cramon P, Hegedus L, et al. The thyroid-related quality of life measure ThyPRO has good responsiveness and ability to detect relevant treatment effects. J Clin Endocrinol Metab 2014;99:3708-17.

15. Rasmussen AK, Feldt-Rasmussen UF. Autoimmune thyroiditis--an infectious disease? Ugeskr Laeger 2002;164:5911-5.

16. Sousa PA, Vaisman M, Carneiro JR, et al. Prevalence of goiter and thyroid nodular disease in patients with class III obesity. Arq Bras Endocrinol Metabol 2013;57:120-5.

17. Chen AY, Frankowski R, Bishop-Leone J, et al. The development and validation of a dysphagia-specific qualityof-life questionnaire for patients with head and neck cancer: the M. D. Anderson dysphagia inventory. Arch Otolaryngol Head Neck Surg 2001;127:870-6.

18. Stenton, C. The MRC breathlessness scale. Occup Med (Lond) 2008;58:226-7.

19. Song RH, Wang B, Yao QM, et al. The Impact of Obesity on Thyroid Autoimmunity and Dysfunction: A Systematic Review and Meta-Analysis. Front Immunol 2019;10:2349.

20. Witkowska-Sędek E, Kucharska A, Rumińska M, et al. Thyroid dysfunction in obese and overweight children. Endokrynol Pol 2017;68:54-60.

21. Reinehr T. Obesity and thyroid function. Mol Cell Endocrinol 2010;316:165-71.

22. Zheng L, Yan W, Kong Y. An epidemiological study of risk factors of thyroid nodule and goiter in Chinese women. Int J Clin Exp Med 2015;8:11379-87.

23. Philpott H, Garg M, Balasubramanian S, et al. Dysphagia: Thinking outside the box. World J Gastroenterol 2017;23:6942-51.

24. Richter JE, Rubenstein JH. Presentation and Epidemiology of Gastroesophageal Reflux Disease. Gastroenterology 2018;154:267-6.

25. Dixon AE, Peters U. The effect of obesity on lung function. Expert Rev Respir Med 2018;12:755-67.

26. Biondi B, Palmieri E, Fazio S, et al. Endogenous subclinical hyperthyroidism affects quality of life and cardiac morphology and function in young and middleaged patients. J Clin Endocrinol Metab 2000;85:4701-5.

27. Bianchi GP, Zaccheroni V, Solaroli E, et al. Health-related quality of life in patients with thyroid disorders. Qual Life Res 2004;13:45-54.

28. Jafari A, Campbell D, Campbell B, et al. Thyroid Surgery in a Resource-Limited Setting. Otolaryngol Head Neck Surg 2017;156:464-71.

29. Watt T, Groenvold M, Rasmussen A, et al. Quality of life in patients with benign thyroid disorders. A review. Eur J Endocrinol 2006;154:501-10.

30. Tabriz N, Uslar V, Tabriz I, et al. Quality of life is not affected by thyroid surgery in nontoxic benign goitre in long-term surveillance-A prospective observational study. Endocrinol Diabetes Metab 2020;3:e00115.

31. Bor J, Cohen G, Galea S. Population health in an era of rising income inequality: USA, 1980-2015. Lancet 2017;389:1475-90.

32. Laubach W, Schumacher J, Mundt A, et al. Social class, life satisfaction and health assessment. Results of a representative study of the German population. Soz Praventivmed 2000;45:2-12.

33. Reiher AE, Mazeh H, Schaefer S, et al. Thyroidectomy decreases snoring and sleep apnea symptoms. Thyroid 2012;22:1160-4.

34. Stang MT, Armstrong MJ, Ogilvie JB, et al. Positional dyspnea and tracheal compression as indications for goiter resection. Arch Surg 2012;147:621-6.

Cite this article as: Shires CB, Beckmann N, Klug T, Boughter JD Jr. Quality of life in obese patients after thyroidectomy for goiter. Gland Surg 2021;10(4):1339-1346. doi: 10.21037 /gs-20-441 